\title{
Écritures individuelles et collectives. Déclarer, ne pas déclarer, savoir dire et savoir jouer avec le dispositif
}

Individual and collective writing. Recording adverse events, avoiding records, and playing " above " and " under » organizational scripts.

\section{Anne Mayere et Angélique Roux}

\section{OpenEdition}

Journals

Édition électronique

URL : http://journals.openedition.org/edc/1162

DOl : 10.4000/edc. 1162

ISSN : 2101-0366

Éditeur

Université Lille-3

\section{Édition imprimée}

Date de publication : 1 décembre 2009

Pagination : $57-78$

ISSN : $1270-6841$

\section{Référence électronique}

Anne Mayere et Angélique Roux, "Écritures individuelles et collectives. Déclarer, ne pas déclarer, savoir dire et savoir jouer avec le dispositif ", Études de communication [En ligne], 33 | 2009, mis en ligne le 01 décembre 2011, consulté le 21 avril 2019. URL : http://journals.openedition.org/edc/1162 ; DOl : 10.4000/edc. 1162

Ce document a été généré automatiquement le 21 avril 2019

(c) Tous droits réservés 


\title{
Écritures individuelles et collectives. Déclarer, ne pas déclarer, savoir dire et savoir jouer avec le dispositif ${ }^{1}$
}

\author{
Individual and collective writing. Recording adverse events, avoiding records, \\ and playing " above » and « under » organizational scripts.
}

Anne Mayere et Angélique Roux

1 Pour mieux comprendre 'l'encadrement des façons d'être aux autres lorsque des dispositifs tendent à les formaliser' ${ }^{2}$, cet article propose d'explorer un dispositif ${ }^{3}$ organisationnel qui prend appui sur une composante du système d'information informatisé: en l'occurrence, il s'agit de la gestion des risques en établissement hospitalier, et plus spécifiquement de la gestion des événements indésirables ${ }^{4}$. Ce dispositif est préconisé dans le cadre de l'accréditation des établissements de soins qui conditionne leur possibilité d'exercice, sachant que les critères à remplir sont devenus de plus en plus exigeants au cours des dix dernières années. Il repose sur une incitation faite à tous les personnels de déclarer les incidents susceptibles de mettre en cause la qualité ou la sécurité du service aux patients, ou de soulever des risques pour les personnels et pour l'établissement. Ce dispositif est en phase avec une dynamique plus globale de formalisation des activités de soin (Acker, 1995 ; Sainsaulieu, 2008). Il s'inscrit dans un contexte d'intensification du travail qui renouvelle la question de l'inscription dans des collectifs de travail (Strauss, 1992 ; Dujarier, 2008).

2 Le dispositif est fait de principes, méthodes, modes opératoires, incluant un processus normé de traitement des déclarations (investigation, diagnostic, puis proposition de solutions). Il fait l'objet d'une formalisation au travers de documents, le premier de la chaîne étant constitué de la fiche de déclaration. Sur le terrain de nos observations ${ }^{5}$, la déclaration papier a été informatisée en 2003 avec une caractérisation requise de 
l'événement par sélection d'items dans des menus déroulants, et un espace de libre saisie, le tout sur une application de l'intranet accessible par une icône dès la page d'accueil. L'outillage informatique est bien diffusé dans cette organisation mais la distribution des postes est hétérogène. Ainsi les internes disposent d'ordinateurs portables et les infirmières de postes collectifs en salle de garde. Ces dernières ne sont donc pas assurées de ne pas être interrogées sur leur activité en cours, ou observées, ce qui peut constituer un obstacle à la déclaration.

Dans une situation où les risques sont permanents, «Les écrits de traçabilité ont une fonction monstrative immédiate - il faut saisir le réel à un certain moment, mais ils sont aussi orientés vers le futur. Que faut-il montrer? Quels sont les paramètres descriptifs à retenir ? Pour répondre à ces questions, il faut non seulement maîtriser les connaissances du moment sur les matériaux mais aussi anticiper les savoirs futurs » (Fraenkel, 2001, p 137). Fraenkel relève ici une conséquence de la traçabilité de l'écrit : le poids des mots. Nous mobiliserons ce questionnement sur les formes et cadres de l'écrit pour questionner la façon dont s'opère l'acculturation à la logique du dispositif. Nous verrons ce en quoi il suppose notamment le respect de principes de dépersonnalisation et de décontextualisation partielle de l'événement pour une 'montée en généralité' parfois bien éloignée des affects mobilisés dans l'instant de l'événement.

\section{Montée en puissance de la formalisation via un dispositif informationnel}

\section{Déclarer ce qui n'est pas maîtrisé}

4 L'énonciation au travail permet le partage des tâches, la coordination de l'action et le positionnement des acteurs les uns par rapport aux autres; de manière concomitante, cet écrit au travail qui fait trace de l'activité et de ses aléas peut aussi être perçu comme facteur de répression et/ou de surveillance des capacités de chacun (Fraenkel, 2001; Boussard, 2001; Bazet et de Terssac, 2007). D'autres se sont interrogés avant nous sur la place du document (Latour, 1994; Guyot et Peyrelong, 2006) ou des écrits (Delcambre, 1997; Charasse, 1992; Cochoy et de Terssac, 2000) dans les activités de travail, notamment au travers des démarches qualité. Les écrits dits de travail font partie de ces inscriptions qui orientent l'action, la stabilisent et contribuent à en articuler les différentes composantes. C'est à la fois une ressource et un moyen mobilisé dans l'activité de travail en ce sens qu'il la formate, la structure alors même qu'il est un produit de cette même activité de travail. Les acteurs ont besoin de ces écrits pour exécuter leur tâche mais également pour faire savoir, pour passer le relais.

5 «Ce qu'on ne maîtrise, pas il faut le taire, telle semble être la résolution prise par de nombreux collectifs» (Fraenkel, 2001, p.130). Or, un événement indésirable est forcément, au moment où il se produit, un événement non maîtrisé ; selon les principes du dispositif de gestion des risques, il doit cependant être dit afin de faire état des failles dans les procédures en place. La question se pose également de l'autorité conquise et attribuée dans ce processus de déclarations. Nous mobilisons la notion d'autorité au sens de Taylor qui situe l'autorité dans le pouvoir d'énonciation (Taylor, 2000, p 140). Le dispositif qualité risques postule que tout membre du personnel est autorisé à déclarer, quelque soit par ailleurs son statut dans cet univers où s'échelonne toute une diversité de classements explicites et implicites dans le prestige des professions et des activités 
(Mathieu-Fritz, Bercot, 2008). Toute une dimension du travail de la cellule en charge de la gestion de la qualité et du risque consiste précisément à susciter cette expression et à en devenir porte parole en la validant, à reconnaître cette énonciation comme valide. L'autorité ainsi constituée n'est pas systématique, certaines déclarations ne sont pas validées, et ceci participe manifestement à la crédibilité du dispositif et de la cellule : elle n'est pas seulement porte-voix, elle valide ou non ce qui a été ainsi énoncé.

Il s'agit ainsi de constituer autant d'indices et de propositions d'amélioration des modalités d'organisation et de coordination, donc de travailler sur le 'texte' de l'organisation pour le mettre en cohérence avec les préconisations des textes globaux tels que formalisés par les instances de régulation.

\section{Un primat croissant de l'écrit sur l'oral}

7 L'hôpital, comme nombre d'organisations contemporaines, est soumis à des règles législatives et à des procédures d'accréditation dont le niveau d'exigence et l'étendue s'accroissent. La formalisation des protocoles, des actes et des processus se double d'une obligation de traçabilité. Une telle orientation tend à faire primer l'écrit sur l'oral, en ce qu'il conditionne de plus en plus la mise en œuvre de l'activité et qu'il la légitime. Ainsi, un membre du personnel qui fait part oralement d'un événement indésirable à l'un des membres de la cellule est incité à 'officialiser' sa requête par la voie du formulaire prévu à cet effet.

8 La relation oral-écrit est ainsi repensée, reformulée. L'écrit constitue le 'sésame' qui autorise et légitime une intervention de la cellule, ainsi que la possibilité pour le personnel de se référer à cette inscription au 'registre' des incidents. De même pour l'analyse des événements que met en œuvre la cellule lors de ses réunions hebdomadaires, le compte-rendu oral doit être retranscrit dans des dossiers ou dans des formulaires pour faire état du travail effectué et justifier du passage aux étapes ultérieures. La procédure de traitement des événements indésirables est constituée d'une enquête dans les services, de réunions hebdomadaires de la cellule pour traiter de la suite à donner à chaque cas, et d'une formulation de propositions de solutions soumises aux responsables et autres personnels concernés. Elle est modelée par un processus mêlant étroitement écrit et oral et qui est formalisé par le logiciel dédié de gestion des risques.

Les écrits sont considérés comme outils de légitimation (de la déclaration, des situations de travail et des pratiques, voire de la cellule elle-même via les statistiques concernant le nombre des déclarations reçues et traitées) et passent par des étapes de validation. Ils sont à la fois objet et trace du travail. Ainsi lors des réunions hebdomadaires, il est question de faire avancer les dossiers dans l'application; a contrario, les déclarations qui 'stagnent', pour lesquelles l'investigation n'a pu être achevée ou qui restent sans proposition crédible de solutions, sont autant de traces d'un travail empêché de la cellule qui pèse sur ses résultats, le ratio des déclarations non traitées sur le total constituant l'un des indicateurs-clé de son tableau de bord. Se fait jour ici une tension interne au dispositif en ce qu'il est orienté vers la sélection des déclarations pertinentes pour mettre en œuvre un travail d'organisation collectif, mais qu'il comporte également ses règles d'enregistrement et de traitement systématiques. Ces règles, dont la prégnance est en bonne part soulignée par le workflow du logiciel dédié, impriment une autre logique, celle qui consiste à tenir des délais dans le travail des déclarations elles-mêmes, à viser des taux de résolution élevés, la résolution pouvant consister en un classement sans suite. 
De ce point de vue, les déclarations les plus inconfortables pour la cellule sont celles qui se justifient en termes de qualité ou de risque, mais qui portent sur des pratiques que l'autorité du dispositif ne suffit pas à modifier. L'indicateur des déclarations sans solution est alors comme la trace de cette impuissance à impulser du changement.

\section{Mettre en place des conditions d'acceptation}

10 La mise en place du dispositif de déclaration des événements indésirables vise à assurer l'amélioration de la qualité de service et de la sécurité du patient et des personnels. Elle est préconisée par les dispositifs d'accréditation qui conditionnent la possibilité pour les établissements de soins de mener leur activité. Cette injonction des instances nationales n'a cependant d'efficacité que si elle rencontre des dispositions parmi les personnels concernés. Selon Mucchielli (1994), parce que les différentes catégories de personnel en interaction ne sont que rarement acculturées aux principes et méthodes de management, les problèmes organisationnels et humains qui apparaissent dans les hôpitaux sont interprétés par les parties en présence en termes de causes à effets localisés, dans une approche qui tend à en rester au stade de la description des éléments de dysfonctionnement visibles, selon des perspectives souvent indépendantes les unes des autres. Le programme de déclaration des événements indésirables dénote une volonté d'aller au-delà de ces analyses parcellaires. Il est mis en place dans le projet de tenir une visée plus globale, mêlant veille, analyse et amélioration des processus, et responsabilisation des personnels.

\section{Un cadre contraignant et flou}

11 Le formulaire de déclaration repose sur des menus déroulants, qui oblige le personnel à 'plier' ce qu'il a vu dans des catégories pré-établies. En même temps, tant la déclaration que l'investigation ultérieure maintiennent des espaces et des moments ouverts de libre expression, qui permettent de réintroduire le ressenti, le caractère situé de l'événement. A l'épreuve des faits, la définition pratique des EI se révèle 'volontairement' floue, ce qui permet des aménagements et des évolutions. Il s'agit de maintenir la vigilance sur de nouveaux risques susceptibles d'advenir tout en trouvant des articulations plurielles sur les motivations du personnel.

12 La fiche de déclaration des EI (cf.annexe 1) est l'aboutissement d'une réflexion collective impliquant des médecins et des cadres de plusieurs services, « pour avoir un panel de la maison. [...] Pour moi, ce n'était pas évident de voir ce qu'on pouvait déclarer. Pour les médecins non plus d'ailleurs » (responsable cellule Qualité Risques Accréditation, QRA). Les membres de la cellule constituée d'un médecin, d'un qualiticien, d'une assistante et d'un membre de la CME ${ }^{6}$ se sont beaucoup interrogés sur le choix des mots. Lors de l'élaboration de la fiche de déclaration, qui s'est inspirée de précédentes fiches utilisées dans d'autres établissements, l'item «erreur de prescription » est devenu très vite "prescription non-conforme ", et le mot " erreur " a été remplacé ailleurs par " défaut de ». Un des principes-clé de la démarche consiste à écarter l'accusation des personnes et les jugements culpabilisants pour interroger les dysfonctionnements au plan de l'organisation; il importait dès lors que les mots utilisés soient autant d'invitations à une telle évolution de perspective. 

était accompagnée d'une notice d'explication de la démarche dans laquelle il était précisé que la personne déclarante était toute personne concernée, c'est-à-dire celle qui avait ou aurait pu subir un préjudice ou en avait été le témoin.

Les définitions qui apparaissent dans les documents de formation et d'information des personnels tendent à décliner une variété de situations, ce qui fait apparaître en creux l'ampleur du territoire et ses indéterminations :

- accident : événement entraînant un dommage,

- incident : événement n'entraînant pas de dommage,

- situation à risque : dysfonctionnement qui aurait pu se traduire par un accident ou un incident,

- situation de non-qualité : dysfonctionnement pouvant entraîner un défaut de confort ou de satisfaction.

Sur six années de fonctionnement, 5067 fiches ont été reçues, ce qui représente un volume conséquent de déclarations, sachant que les professionnels de santé, praticiens, infirmiers et cadres soignants sont les principaux déclarants. Un débat récurrent au sein de la cellule vise à déterminer celles des déclarations qui concerneraient de 'vrais' événements indésirables, en mettant de côté des situations de non-qualité peu favorables pour l'appréciation des patients mais qui sont considérées comme 'non susceptibles d'entraîner des dommages'. Dans le traitement qu'elle fait des déclarations, la cellule tente de les catégoriser au regard de ces critères. Les courbes ainsi tracées à partir des indicateurs dessinent ce que la cellule interprète comme une modification au fil du temps des types d'événements déclarés, qui irait dans le sens d'une part croissante des situations à risques, incidents et accidents, traduisant donc une acculturation croissante au dispositif. Pour autant, il ne s'agit pas d'empêcher des déclarations qui échappent au critère du dommage, effectif ou potentiel. "C'est aussi important de les avoir (les autres déclarations), parce qu'elles peuvent devenir des situations de non-qualité si elles perdurent.» (Responsable cellule QRA). L'absence d'une définition précise et surtout admise par tous conduit les membres de la cellule, au cours des réunions hebdomadaires, à s'interroger sur certains cas, et engendre régulièrement des discussions vives. Il existe une tension irréductible entre une approche rationalisante qui voudrait objectiver les critères, et une impossible clôture du territoire des indésirables, sachant qu'il ne s'agit pas seulement de qualifier ce qui est advenu, mais ce qui aurait pu advenir, et qu'il n'existe pas de délimitation stricte entre dommage et non-qualité.

Travailler à partir de définitions très larges et pouvant recouvrir des événements très divers a été un choix d'orientation initiale. Il s'agissait de promouvoir un signalement large et banalisé, concernant 'tout événement imprévu ayant entraîné un dommage ou sans conséquence' (document interne, 2005). Au fil du temps, la cellule a essayé d'infléchir cette orientation tout en rendant compte de l'ambivalence d'une démarche qui vise à susciter en premier lieu l'adhésion des personnels:

« depuis un an ou deux, on dit aux gens quand ce n'est pas de la gestion des risques, que c'est pas pour nous. Mais au début, on n'osait pas parce qu'en fait ce qui était important c'est que les gens déclarent. (...) L'important c'est de bien comprendre qu'un événement indésirable c'est souvent le fait d'un enchaînement de plusieurs actions qui créent des petits dysfonctionnements, mais qui au final peuvent donner lieu à des situations graves. C'est une affaire collective. » (Responsable cellule QRA)

Études de communication, 33 | 2009 
17 Malgré le surcroît de travail qu'engendrent des déclarations hors cadre, il n'a pas été retenu d'évoluer vers une définition plus restrictive. Toutefois, la cellule nuance les événements à prendre en compte en fonction de l'importance à leur accorder : il s'agit en quelque sorte de s'accorder sur ce qui va faire autorité.

«Certains trouvent des cas importants, d'autres moins. C'est souvent en fonction de l'expérience de chacun, de la prise en compte des malades, selon qu'on est plus ou moins proche d'eux. [...] Il n'y a pas d'homogénéisation totale possible, même si on tente de réduire les écarts. Il y a des gens qui nous disent avoir vécu des situations normales pour eux, mais qui auraient du être déclarées. » (idem)

Pour d'autres membres de la même cellule, l'événement indésirable est celui qui modifie le chemin clinique du patient, son parcours au sein de l'institution et de ses procédures. La difficulté dans l'évaluation de la criticité de tel ou tel cas est lié à l'effet de contexte, et semble se construire dans la discussion. Les réponses pré-établies ne suffisent pas toujours à exprimer un événement dans sa singularité et sa complexité. Par ailleurs, selon les individus, la perception d'un item n'est pas forcément identique; concernant la gravité de l'événement, les déclarants ne mobilisent pas nécessairement les mêmes références pour en juger. L'espace réservé à la description du cas est donc particulièrement important dans la mesure où il permet au déclarant d'exprimer les particularités de l'événement survenu, de le contextualiser. C'est aussi ce qui fait l'importance de l'investigation ultérieure.

\section{Un lieu et une occasion d'échanges}

Dans une situation où l'on observe plus des processus, des dynamiques d'appropriation et d'acculturation que des manières de faire, on peut considérer que les acteurs, à travers leurs pratiques face aux écrits au travail (re)trouvent un espace de liberté. Certes le cadre est contraignant, le formatage de l'énonciation est conséquent, mais l'apprentissage collectif du dispositif et les formes d'interaction qui se sont développées avec les membres de la cellule ont permis aux personnels d'identifier de possibles jeux dans et sur les cadres. Ainsi, pour les événements indésirables, la définition mais également les consignes sont floues sur ce qui peut être déclaré ou non afin de laisser aux acteurs (qu'il s'agisse des déclarants ou de la cellule) une marge de manœuvre. Cela autorise une action de veille autour d'aléas qui, par définition, ne sont pas définissables. Cette manière de faire permet également une autre forme d'adhésion au dispositif : la déclaration 'soupape'.

Dans ses premières années d'existence, les bureaux de la cellule étaient localisés dans le voisinage immédiat de ceux de la direction de l'établissement, manifestant l'importance attribuée à la démarche tout en facilitant les échanges à ce niveau. Ils ont été ensuite déplacés dans un autre étage, ce qui s'est avéré, à l'épreuve des faits, présenter d'autres avantages. En effet, alors que les membres du personnel visitaient rarement les bureaux dans l'étage directorial, ils sont beaucoup plus nombreux à passer par les bureaux actuels, et en ont fait ce que les membres de la cellule désignent en plaisantant comme une «petite Suisse » :

«[...] il y a beaucoup beaucoup de mouvement, beaucoup de gens qui viennent, qui téléphonent, qui racontent des histoires. Ils savent qu'ici c'est la Suisse: ils viennent, ils rentrent, ils vivent, ils ressortent le cœur léger.» (assistante cellule QRA). 
« Des fois je n'ai le temps de rien dire. Ils ont tellement besoin de livrer leur stress

qu'ils se déversent autour de l'événement. » (idem)

21 conditions d'acceptation du dispositif afin que soit déclaré ce qui n'est pas maîtrisé. Mais elle intervient également au plan des modes d'appréhension des phénomènes concernés. La genèse d'une déclaration tient généralement à des configurations de situations, à des personnes, à des services ou des groupes professionnels. Les déclarations sont ancrées dans des parcours individuels et collectifs, au sein d'une organisation qui facilite leur expression hors d'un ancrage trop spécifique à la notion de qualité. Il faut toutefois que dans la narration de l'événement soient respectés des principes de dépersonnalisation et de décontextualisation qui permettront une généralisation. En cela l'acculturation au dispositif ne passe pas seulement par son acceptation mais par l'intégration d'un cadre de perception et de formulation.

\section{Sources et re-sources : de l'oral à l'écrit. Un travail de dépersonnalisation par la formalisation}

Les personnels rencontrés font état du caractère contraignant de ces écrits spécifiques via le formulaire informatisé. Pour autant, ce travail qui est plus de catégorisation que de formulation est accepté en raison de ce qu'il permet d'initier. D'une part il est démontré que ceci déclenche une action de la cellule, à savoir dans un premier temps, une investigation, un 'travail d'enquête'. D'autre part cette investigation donne lieu à des échanges oraux, en face à face, qui permettent de redonner sa densité, sa singularité à l'événement, de faire une certaine place aux affects, voire d'échanger sur les solutions envisageables ou souhaitables.

Cet oral n'en est pas moins fortement cadré, au sens où il est structuré par des méthodes (recherche de causes et de conséquences, diagramme d'Ishikawa, reconstitution des enchaînements d'actions ou analyse de causes selon le schéma 'Reason'...). Ces méthodes ont en commun de mettre de côté les questions de personnes, et de chercher à identifier ce en quoi cet événement singulier peut mettre à jour des dysfonctionnements de l'organisation. En cela, l'ensemble des écrits et oraux textualisés, qui contribuent à la gestion des événements indésirables, peut être envisagé comme participant à la production d'une ressource informationnelle au plan de l'organisation. Ces productions d'informations tant dans la formalisation première via le formulaire que dans les traitements ultérieurs reposent sur des cadres spécifiques, qui sont assez éloignés des réactions spontanées des personnels concernés, et qui de proche en proche visent à les acculturer à cette façon de voir et de penser. En mobilisant la notion de re-source telle que développée par Levitan, nous allons explorer plus avant le processus à l'œuvre.

\section{Des sources individuelles à une re-source organisationnelle}

Karen B. Levitan (1982) s'est attachée à décrire un cycle de vie de la production de l'information. L'utilisation de ce terme laisse entrevoir la possibilité d'étapes réparties dans le temps et observables en tant qu'unités évolutives. 


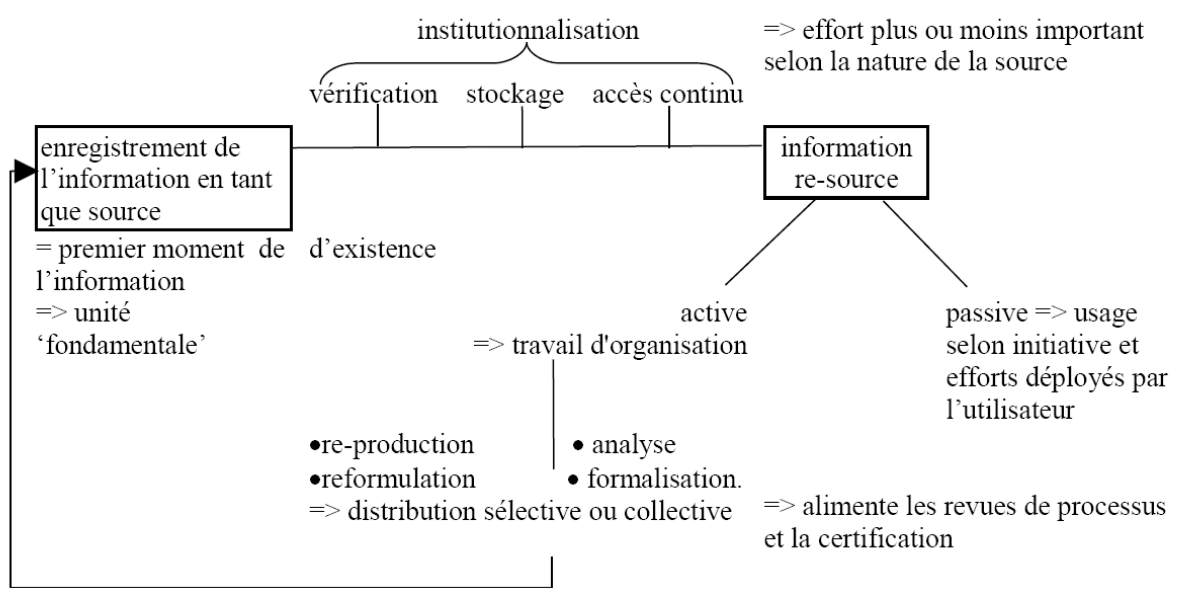

Ce schéma a été conçu dans le cadre de travaux sur les produits informationnels et la marchandisation de l'information. Bien que le contexte de notre analyse soit différent, il permet d'identifier des points-clés liés à la mise en place et au fonctionnement du dispositif de déclaration des événements indésirables. Le dispositif étudié a en effet pour objectif premier la production d'une information pertinente, qui puisse être mobilisée par des tiers dans la même organisation, pour participer à un travail visant à ajuster les processus et les modes de coordination aux exigences ainsi identifiées. Il permet de passer de sources contextualisées chargées d'affect à des re-sources, avec une montée en généralité qui contribue au travail d'organisation en sortant l'événement de sa seule dimension située. Les déclarations sont écartées ou précisées, retravaillées, spécifiées, déjouées et rejouées, jusqu'à devenir des re-sources autorisées qui vont impulser tout un travail d'organisation, comme en témoigne le cycle de traitement des événements indésirables représenté ci-après. 
ANNEXE 2

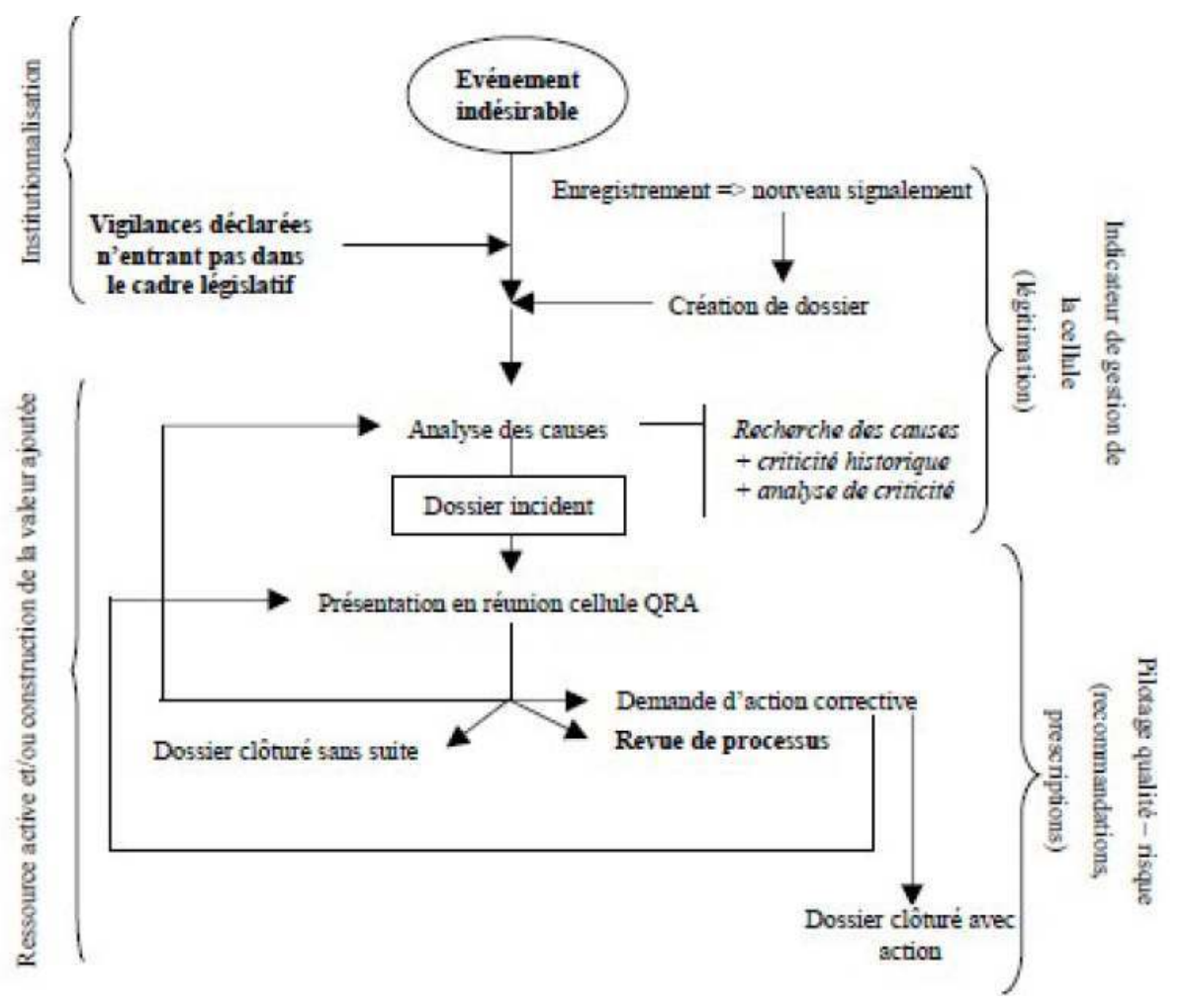

En matière d'événement indésirable, le passage d'une source à une re-source suppose 'd'alléger' l'information de sa charge d'affects et de son articulation sur des personnes. Il ne s'agit pas en effet de mettre en cause les personnes engagées dans l'événement indésirable, de les qualifier en 'victimes' ou 'coupables', mais de questionner ce en quoi l'organisation s'est avérée défaillante. En cela, le travail du dispositif est conçu de façon à éviter le passage sur un registre du signalement, ce qui est désigné et craint comme relevant de la délation par le personnel. La grille de déclaration focalise l'expression sur la caractérisation de l'événement. Elle soumet des choix en référence à des situationstypes. Le déclarant doit dès lors s'astreindre à trouver les items les plus proches de l'événement singulier dont il veut rendre compte, quitte à les combiner ou à arbitrer entre ceux qui en rendraient le mieux compte. Ce travail de sélection constitue une première forme de montée en généralité que la cellule continue à opérer au fil de son investigation. Ce qui peut être de l'ordre d'un 'pré-jugé' du déclarant est ainsi mis en discussion, mis à l'épreuve pour être ou non constitué en information pertinente pour la collectivité.

On peut dans ce processus s'interroger sur l'écrit (qui même s'il est individuel devient par le biais des procédures, collectif) comme inscrit dans une dynamique de dépersonnalisation / repersonnalisation.

" Le passage de la parole à l'écrit, du propos singulier au texte collectif, la circulation des écrits dans une équipe, une entreprise ou une organisation sont source de désordres, drainent des conflits de soi à soi, de soi aux autres [...]. Même dans des situations d'écriture très contraintes, de textes très normés, la subjectivité n'est jamais totalement évacuée, même si c'est, bien souvent, sous des formes qui l'aliènent. » (Dumont \& Revuz, 1994, p.11). 
Ainsi les déclarations peuvent-elles exprimer des dissensions fortes sur des actes médicaux; elles peuvent venir traduire l'exaspération d'un secrétariat médical qui apprend encore une fois à la dernière minute l'absence de tel médecin alors que les confirmations de rendez-vous de consultation sont déjà parties au courrier ; elles peuvent aussi faire état de ce qui est identifié comme la manifestation du non respect d'un domaine d'intervention de spécialistes dans l'enchaînement des interventions autour des patients et de leur dossier. Autant d'exemples dans lesquels interviennent des affects, des conflits, des rivalités, qui vont trouver en se pliant dans ces écrits une forme audible au plan de l'organisation pour autant que ces déclarations passent les épreuves de leur confrontation 'aux faits', qu'elles puissent résister devant une investigation qui écarte les conflits inter-individuels pour interroger la possible défaillance des processus organisationnels.

C'est en effet la cellule par son travail d'investigation et de traitement qui va ou non fonder l'autorité attribuée à ces déclarations, en les endossant, en s'en faisant porteparole, en se constituant ainsi comme macro-acteur autorisé à intervenir en ce qui concerne la gestion des risques. Les déclarations sont anonymes, pour respecter la confidentialité et inciter tous les personnels à déclarer, quelles que soient leur situation hiérarchique ou celle du collectif dans lequel ils sont insérés. Selon les principes posés dès les débuts de la démarche et tels que retranscrits dans un document interne, 'la cellule distingue les situations signalées qui doivent faire l'objet d'une description détaillée et d'une analyse des événements qui ont conduit à l'incident' (document interne, 2005). Suite à cette investigation, le 'classement sans suite' est une des décisions susceptible d'être prise. La cellule a ainsi un rôle de filtre important qui fait aussi partie de ce qui la légitime.

«Et là, si vous avez vu Mme (responsable cellule QRA), elle aussi fait le tri avec l'équipe de la gestion du risque, elle dit voilà ça c'est pas significatif, on se plaint qu'il avait une chaussette noire au lieu de blanche, d'accord, ce qui arrive quelque fois, euh, mais en revanche voilà quelque chose de significatif on va demander de l'information en aval, ça pourrait être infiniment plus grave aussi et justifier une vraie enquête. » (médecin chef de service)

30 Ainsi le travail de vérification de la cellule va ou non attribuer une autorité aux déclarations transmises en les labellisant, en les certifiant, après les avoir passées au crible des faits objectivables, et soumis aux controverses des personnels concernés. Le passage d'une source à une re-source s'opère donc au travers d'une série de cadrages et de vérifications : dès l'enregistrement de l'information, le script organisationnel spécifie ce qui peut être déclaré et les critères selon lesquels il peut en être fait état; l'investigation met à l'épreuve et prolonge cette mise en forme en se focalisant sur ce qui peut contribuer au travail d'organisation.

\section{Mise à l'épreuve et chaîne de traitement des déclarations}

Suite à leur transmission, les fiches de déclarations sont traitées par la cellule QRA. Une première investigation vise à identifier les caractéristiques de l'événement déclaré, à en repérer les causes au plan des processus organisationnels et à consolider l'argumentaire sur les conséquences potentielles; ensuite, la cellule décide collectivement au cours d'une réunion hebdomadaire des suites à donner, des actions à engager. Le processus de traitement des événements indésirables se matérialise à travers un certain nombre de documents, de la déclaration par les opérationnels aux rendus et demandes d'action 
émanant de la cellule qualité, en passant par les documents d'analyse internes à cette même cellule. Si ces documents ont a priori des rôles différenciés, s'ils interviennent à des moments précis du processus de traitement des événements indésirables, chaque acteur y injecte non seulement de l'information mais également - à travers ses façons de faire (ou de ne pas faire) - du sens. Le choix du support de la déclaration donne lui aussi un sens à cette déclaration. Mobiliser le support, le formulaire de déclaration des EI, c'est déjà en soi un message d'urgence quand on a assimilé que la fiche est un déclencheur de l'attention de la cellule.

La déclaration des EI suppose de qualifier la gravité et les conséquences de l'événement déclaré. Cette qualification par les déclarants est de proche en proche confrontée aux catégories établies par la matrice de criticité 7 à laquelle se réfère la cellule afin de servir de base de décision, et qui est utilisée pour la codification des cas à des fins statistiques.

Les formations des nouveaux arrivants, et les supports d'information diffusés par la cellule, visent à alerter le personnel et à l'inciter à participer. Il s'agit de faire de chacun une 'sentinelle', un déclarant à même de réinterpréter et de décontextualiser l'EI, et de rendre compte de cet événement singulier vécu dans une situation de travail, en choisissant des items dans un menu déroulant préformaté.

Le cadrage du contexte est également en partie imposé par la trame temporelle de la déclaration. En effet, le moment de survenue de l'événement et le moment du traitement étant décalés dans le temps et amenant des acteurs différents à intervenir, obligation est faite de donner à la cellule quelque chose d'interprétable en dehors de l'action: ce que Fraenkel désigne comme les écrits cadrés par des éléments de contexte. L'événement indésirable résiste-t-il au-delà de son instantanéité, peut-il être délocalisé? Ainsi, le support oblige à expliciter une partie du contexte et contribue à ce qu'il perdure au-delà de la situation. Cette façon de faire est d'autant plus importante que Fraenkel (2001, p.126), se référant à l'approche linguistique de Derrida (1967) insiste sur ces situations de communication différée où, par l'absence du déclarant, «l'écrit ne semble jamais être redevable de quelque situation que ce soit». Un écrit situé permettra alors la construction de sens à travers des éléments situationnels. Ces éléments situationnels peuvent faire l'objet de différentes interprétations, et se posera alors la question de la construction de sens a posteriori. Mais ce décalage dans le temps est aussi un temps laissé au déclarant pour prendre le recul nécessaire à la décontextualisation et surtout à la dépersonnalisation. Il offre aussi une marge pour construire une stratégie: une forme d'acculturation positive.

\section{Un jeu pluriel dans les cadres et sur les cadres}

Les déclarations sont faites en majorité par les personnels soignants (praticiens, infirmières, aides-soignantes). Tant le nombre des déclarations que leur caractère relativement diffus permet d'avancer qu'une certaine appropriation de la démarche a bien eu lieu, du moins dans ces catégories de personnels.

\section{La cellule comme 'porte-voix'}

Si la démarche s'est diffusée, elle reste inégalement pratiquée. En parallèle, des îlots de non-déclarants s'organisent, îlots qui en retour font l'objet de déclarations rédigées par 
les services avec lesquels ils sont amenés à être en relation. C'est le cas par exemple du service de stérilisation à l'égard du bloc opératoire. Régulièrement, et malgré les procédures et moyens mis en place (boîtes hermétiques pour les aiguilles et les lames, bacs de récupération pour les autres matériels), les personnels du service de stérilisation trouvent des objets tranchants dans les bacs, avec le risque de prendre ces objets à pleine main dans la mesure où ils sont censés ne trouver que des instruments inoffensifs (pinces ou écarteurs). Le risque de blessure voire d'infection est considéré comme important. Une stratégie a été mise en place au sein du service stérilisation par sa responsable, en coordination avec la cellule, afin de déclarer systématiquement tout incident lié au bloc opératoire. Il s'agit de (re)créer le collectif pour se faire entendre, d'utiliser la cellule QRA comme porte-voix et ainsi, de reprendre l'initiative : «En déclarant on donne du poids aux plaintes ", « en donnant des éléments la cellule qualité peut en parler à la direction ", " ça servait à rien de râler dans son coin parce que quand on râlait dans son coin, on n'avançait pas» (personnels du service stérilisation). Un travail d'institutionnalisation s'opère au fil de la mise en forme des déclarations et de leur officialisation, de leur autorisation par la cellule; il permet de ré-équilibrer au moins en partie les rapports de pouvoir entre services, entre personnels, en relayant le problème perçu localement par une parole autorisée et en le recadrant comme dysfonctionnement organisationnel.

\section{Ecrits chuchotés ou clamés à la cantonade}

$\mathrm{Au}$ travers des entretiens auprès des personnels et des observations de réunions de la cellule qualité risques, des situations-types de déclarations ont été dégagées (Bazet et al., 2007). Nous retiendrons plus particulièrement ici celles qui mettent au travail les rapports entre écrit et oral, et entre individus et collectifs. A ces situations s'articulent diverses modalités de déclaration: on peut déclarer discrètement, ou l'annoncer à la cantonade, voire recruter d'autres déclarants. Un même déclarant peut selon les situations s'exprimer selon tel ou tel registre, et ce sont ces configurations de déclaration qu'il est apparu plus particulièrement pertinent de spécifier.

Le fait que la déclaration soit écrite permet de faire trace selon des modalités fort variables: trace discrète et dont l'anonymat est valorisé lorsque l'initiative est individuelle et que la situation hiérarchique ou la pression du collectif ne permettent pas d'en faire état publiquement; trace collective et concertée lorsque le dispositif est mobilisé pour contester une décision hétéronome. Dans ce dernier cas, le dispositif de déclaration des EI est utilisé pour produire un argumentaire, faire valoir le point de vue du personnel au regard de décisions prises dans d'autres instances et qui sont considérées localement comme aberrantes compte tenu des contraintes du service. Les déclarants se saisissent alors de 'l'étendard du patient' pour faire valoir les intérêts qui lient patients et personnels, par exemple autour de ce qui est désigné comme un sous-effectif infirmier. La trace écrite est ici appréciée en ce qu'elle 'massifie' la plainte, qu'elle permet en recrutant d'autres déclarants d'instaurer une forme de pétition virtuelle, en prenant au mot le dispositif et sa rigueur d'enregistrement: toute déclaration est tracée, elle incrémente des indicateurs, et il est possible d'y faire référence dans d'autres contextes.

L'écrit peut aussi être envisagé comme recours préventif en cas de contentieux, dans ces cas difficiles pour lesquels le patient devient source de risques, qu'il devient agresseur, au travers notamment de formes plus ou moins avancées de harcèlement moral. Déclarer, alors, c'est être assuré de ce qu'une investigation indépendante sera menée rapidement, 
tant qu'il y a des témoins et des traces, selon des critères objectivés et des méthodes reconnues comme valides, et donc qu'il sera possible de s'y référer si les patients s'adressent à la direction ou à une instance de médiation.

40 L'acculturation au dispositif se traduit dès lors par un jeu pluriel, individuel et collectif, qui opère selon le cas dans le cadre et sur le cadre ainsi posé (Latour, 2007). Jouer sur le cadre consiste notamment à chercher à faire dire et faire faire au dispositif plus ou autre chose que ce que délimite sa fonction première, à savoir gérer les risques. Il peut ainsi s'agir de faire état, via la cellule, de contradictions ou tensions auprès des instances de direction ; il est alors fait référence aux valeurs promues par le dispositif, la sécurité et la qualité du service aux patients, pour proposer d'autres interprétations des contraintes et enjeux. C'est ainsi tirer partie de la rationalisation organisationnelle à laquelle participe ce dispositif pour en contester certaines dimensions, tels celles qui interviennent dans la limitation des ressources et dans l'intensification du travail.

\section{Ecrire dans des limites et aux limites}

41 Ce dispositif peut être perçu comme dangereux pour les collectifs, pour la solidarité requise dans une coordination de tous les instants, lorsqu'il pointe sur les personnes. De ce point de vue, le personnel s'avère déclarer dans des limites et aux limites. Tout ne se déclare pas, y compris des événements qui relèveraient pourtant typiquement des 'indésirables'. Le personnel va alors chercher à réguler localement, quitte à manifester son acculturation au dispositif en en reprenant les méthodes («je mène ma propre enquête" nous dit une infirmière) mais en adressant la question aux personnes impliquées, c'est-à-dire en ne construisant pas la distance entre l'événement et les personnes. Une autre modalité consiste à faire en sorte que la déclaration soit effectuée par un service adjacent directement ou indirectement concerné par l'événement, de façon à remonter en généralité par la désignation d'un problème à l'articulation de deux services, qui soude chaque collectif dans l'analyse de ses propres processus et défaillances éventuelles.

Lorsque le dispositif est considéré comme dangereux pour le collectif, diverses modalités vont donc chercher à circonscrire le danger, y compris celle qui consiste à ne pas déclarer, à « laver le linge sale en famille ». Ce même dispositif peut aussi être saisi pour mobiliser le collectif et lui permettre d'exprimer d'autres priorités, d'autres points de vue sur l'organisation, ses missions, ses valeurs, son pilotage et ses modes de gestion du changement.

\section{Conclusion}

43 A travers ces pratiques de déclaration des événements indésirables se constitue une forme de réflexivité organisationnelle entre des collectifs aux contraintes différentes, réflexivité matérialisée par la création d'un référentiel commun en bonne part implicite sur ce qui se déclare, et comment. Ce dispositif pourrait paraître à première vue comme extrêmement contraignant, au travers des cadres qu'il impose tant en termes de déclaration que de traitement et de suivi. Or nos observations font apparaitre une dynamique faite d'échanges écrits et oraux, individuels et collectifs, mobilisant en complémentarité une combinaison d'autres dispositifs (comités morbidité mortalité, groupes d'analyse de processus, dispositifs de vigilance plus spécialisés - hémo-vigilance, 
pharmaco-vigilance, etc.). Le flou sur les territoires de l'indésirable, dans cet entrelacs de territoires dédiés que dessine la règlementation, empêche de clôturer le dispositif et laisse par là même place à des interprétations plurielles. Différents espaces de parole cohabitent permettant de mettre au travail un même événement selon différentes perspectives, par exemple celle des pratiques médicales en comité des complications, et celle des dysfonctionnements organisationnels en cellule qualité risques. Le caractère proliférant et flou du dispositif, cette juxtaposition d'écrits peuvent apparaitre bien éloignés de la rationalisation tant sollicitée des organisations de santé et des professionnels du soin. Notre interprétation est qu'ils conditionnent la réussite relative du dispositif et la dynamique d'acculturation, dans la mesure précisément où sont ainsi implicitement reconnues la pluralité des logiques, les contradictions intrinsèques entre logiques professionnelles et logiques gestionnaires, et où diverses formes de régulation peuvent être cherchées et temporairement trouvées dans ces marges.

\section{BIBLIOGRAPHIE}

Acker, F., (1995), « Le travail de formalisation de l'activité infirmière. Gérer les soins, conduire les pratiques ", 61ème Congrès annuel de l'Association Latine pour l'analyse des systèmes de santé, p 195-201.

Bazet, I., Mayère, A., Jolivet, A., Roux, A., Cattla, M., Simbille, J., (2007), Conception et appropriation d'un dispositif de gestion d'événements indésirables - Confiance, défiance et territoires de l'indésirable, rapport CERTOP - LERASS pour la MiRe DREES, Décembre, 139p.

Bazet, I., de Terssac, G., (2007), La rationalisation dans les entreprises par les technologies coopératives, éd. Octarès.

Borzeix, A., (1998), « Comment observer l'interprétation? » in Borzeix A., Bouvier A., Pharo, P., Sociologie et connaissance. Nouvelles approches cognitives, Paris : CNRS Editions, p. 177-194.

Boussard, V., (2001), « Quand les règles s'incarnent. L'exemple des indicateurs prégnants », Sociologie du Travail, vol. 43, n43, p. 533-55.

Charasse, M., (1992), «L'usine, l'écriture et la place », Genèse, n7, mars 1992, p.63-93.

Cochoy, F., de Terssac, G., (2000), « Au-delà de la traçabilité : la mappabilité. Deux notions connexes mais distinctes pour penser les normes de management », in La production des normes entre État et société civile, Serverin, Évelyne Berthoud, Arnaud (dir.), Paris, L'Harmattan, 2000, p. 239-249.

Cooren, F., (2004), “Textual Agency: How Texts Do Things in Organizational Settings”. Organization, 11(3), p. 373-393.

Delcambre, P., (1997), Ecriture et communications de travail. Pratiques d'écriture des éducateurs spécialisés, Lille : Presses Universitaires du Septentrion.

Derrida, J., (1967) De la grammatologie, Paris : Editions de Minuit. 
Dujarier, A.M., (2008), « 'Prendre sur soi' : l'individualisation du travail d'organisation », in G. de Terssac (dir.), La précarité : une relation entre travail, organisation et santé, éd. Octarès, $\mathrm{p}$. 107-118.

Dumont, M., Revuz, C., (1994), « A qui profite l'écrit. Editorial », Education permanente, n¹20 (3), novembre-décembre 1994, p.7-12.

Fraenkel, B., (2001), « La résistible ascension de l'écrit au travail », in Borzeix A., Fraenkel, B., (éd.), Langage et travail. Communication, cognition, action, Paris : CNRS éditions, p. 113-142.

Guyot, B., Peyrelong, M.-F., (2006), « Le document dans une perspective organisationnelle. Un objet comme les autres? », Sciences de la Société, n68, mai 2006, p.45-59.

Latour, B., (1994), « Une sociologie sans objet ? Remarques sur l'inter-objectivité », Sociologie du travail, vol.36(4), octobre 1994, p.587-607.

Latour, B., (2007), « Résumé d'une enquête sur les modes d'existence ou Bref éloge de la civilisation qui vient », Colloque de Cerisy, juin, 238p.

Levitan, K.B., (1982), «Information resource as goods in the life cycle of information production ", Journal of the American Society for Information Science, vol.33 (1), Janvier 1982, p.44-54.

Mathieu-Fritz, A., Bercot, R., (2008), Le prestige des professions et ses failles, Paris : Hermann éditeurs, 313p.

Mucchielli, A., (1994), « Soigner l'hôpital », Sciences humaines, n44, novembre 1994.

Roux, A., (2004), "Système d'information et dispositif informationnel : quelle articulation ? ", Sciences de la Société, ${ }^{\circ} 63$, octobre 2004, pp.27-40.

Sainsaulieu, I., (2008), (dir.) Les cadres hospitaliers : représentations et pratiques, éditions Lamarre

Strauss, A., (1992), La trame de la négociation. Sociologie qualitative et interactionnisme. Ed. L'Harmattan, 320p.

Taylor, J., Van Every, E., (2000), The emergent organization, Communication as its site and surface, Lawrence Erlbaum Associates Pub.

\section{NOTES}

1. Les auteures remercient les évaluateurs de cet article pour leurs remarques et suggestions, dont ils trouveront la trace au fil de ces lignes, tout en gardant la pleine responsabilité de cet écrit.

2. Cf. l'appel à contribution pour ce dossier.

3. Dans cet article, la notion de dispositif met en avant un utilisateur certes cadré (par le système d'information, par des procédures) mais également envisagé dans ses dimensions autonomes (dans la mobilisation qu'il peut faire du dispositif), l'expression système d'information désignant plus communément la combinaison des outils techniques (Roux, 2004).

4. Cet article est une valorisation des matériaux rassemblés et analyses développées dans le cadre d'un programme de recherche associant le CERTOP et le LERAS, programme PCCD/GEI qui a bénéficié du soutien de la MiRe/DRES, et de la $\mathrm{R}$ égion Midi-Pyrénées. $O \mathrm{nt}$ participé à ce programme I. Bazet (responsable scientifique), A. Mayère, A. Roux et J. Simbille, LERAS, équipe CHOCQ, et M. Cattla, CERTOP, Université de Toulouse. 
5. Pour cette recherche, nous avons retenu une méthodologie de type compréhensive. Premièrement, nous sommes partis des écrits, des énoncés, des traces pour comprendre les rationalités qui sous-tendent le travail ayant donné lieu à l'élaboration de la quantification et du repérage des événements, à la définition du circuit de déclaration et à leurs évolutions. Ensuite 39 entretiens semi-directifs ont été menés : auprès des membres de l'équipe en charge de la gestion des événements indésirables, auprès de membres du personnel, dans un premier temps en suivant le parcours du patient, puis au sein d'un même service et d'un service limitrophe. D'autre part, nous avons observé 18 réunions de gestion des événements indésirables, et 6 réunions de processus.

6. Commission Médicale d'Établissement rassemblant des praticiens médecins et professeurs praticiens hospitaliers, principale instance médicale auprès de la direction de l'établissement.

7. Cette matrice à deux entrées met en avant des critères de gravité et de fréquence, évalués sur une échelle de 1 à 4 , les risques étant qualifiés d'insignifiants, acceptables, modérés ou inacceptables.

\section{RÉSUMÉS}

Les déclarations d'événements indésirables dans les établissements hospitaliers font l'objet de formalisation au travers de documents, dont le premier est la déclaration que chaque membre du personnel est convié à remplir dès lors qu'il a assisté à un incident ou quasi incident susceptible d'entraîner des dommages ou des défauts de qualité pour les patients, pour le personnel ou pour l'institution. Cet article explore les conditions d'acceptation par les personnels de cette mise en écrit du non-maitrisé. Une fois ces déclarations enregistrées, le travail de la cellule Qualité Risques Accréditation consiste à mettre à l'épreuve ces déclarations et les "autoriser " en ce qu'elles peuvent être des indices de défaillances organisationnelles. Au fil de son appropriation par le personnel, ce dispositif fait l'objet d'un jeu pluriel dans et sur les scripts organisationnels (Latour, 2007), jeu qui permet l'expression de logiques différentes.

Risk management in hospitals relies on the formalization of documents, and first of all on the adverse event recording. Every employees are suppose to report on errors, mishaps or "critical incidents', nowadays through an intranet dedicated software. In this paper, we identify conditions for the acceptance of such an adverse events reporting by the employees. Once the report transmitted, the activity of the risk management team consists of submitting this record to inquiries, assessing its'authority', according to a search for the related organizational defaults. Employees' appropriation of such methods and approaches sustains the development of different recording situations, following trajectories where they can be successively 'above' and 'under' the organizational scripts (Latour, 2007).

\section{INDEX}

Mots-clés : écrits de travail, formalisation, scripts organisationnels, appropriation, rationalisation, risques, qualité

Keywords : professional writing, rationalization, formalization, risk management, quality management, organizational scripts 


\section{AUTEURS}

\section{ANNE MAYERE}

Anne Mayère est professeure à l'Université Toulouse 3, réalise des recherches concernant la rationalisation des organisations, les risques, et le système de santé.

\section{ANGÉLIQUE ROUX}

Angélique Roux est maître de conférences en Sciences de l'Information et de la Communication, et chercheur à l'Université de Toulouse. Après avoir abordé dans son travail de thèse les jeux de réflexivité autour du triptyque concepteurs-utilisateurs-outils, ce sont les questionnements concernant les logiques et les modalités de mobilisation des technologies dans le cadre de la production collective d'informations qui constituent le noyau de son approche. Elle travaille actuellement sur des questions liées au risque et à la santé autour de la gestion des événements indésirables en milieu hospitalier et de l'informatisation du dossier patient. 